\title{
Studies on in vitro release of CPM from semi-interpenetrating polymer network (IPN) composed of chitosan and glutamic acid
}

\author{
K KUMARI and P P KUNDU* \\ Department of Chemical Technology, Sant Longowal Institute of Engineering and Technology, \\ Longowal 148 106, India
}

MS received 2 October 2007; revised 26 October 2007

\begin{abstract}
Interpenetrating polymer network (IPN) beads consisting of chitosan-glutamic acid were prepared for in vitro study of controlled release of chlorpheniramine maleate (CPM). A viscous solution of chitosanglutamic acid was prepared in $2 \%$ acetic acid solution, extruded as droplets through a syringe to alkalimethanol solution and the precipitated beads were crosslinked using glutaraldehyde solution. Swelling and drug release studies were carried out. Transport of release medium through the semi-IPN depended upon its pH and extent of crosslinking. The structural and morphological studies of beads were carried out by using a scanning electron microscope (SEM). The larger surface area of beads as well as their ease of handling makes them ideal agents of controlled release.
\end{abstract}

Keywords. Beads; chitosan; IPNs; release; swelling.

\section{Introduction}

Chitosan is a polysaccharide comprising of copolymers of glucosamine and N-acetyl glucosamine. Chitosan is the deacetylated derivative of chitin, which is one of the most abundant natural polysaccharides containing nitrogen. The primary unit of chitin is 2-acetamido-2-deoxy- $\beta$-D-glucose while that of chitosan is 2-amino-2-deoxy-D-glucose linked by $\beta(1 \rightarrow 4)$ glucosidic linkage (Muzzarelli 1985). Being biodegradable and biocompatible, chitosan has been used in the formulation of particulate drug delivery systems to achieve controlled drug delivery (Thanoo et al 1992; Ko et al 2002). Chitosan is commercially available and can be reshaped into different forms of films, fibres, hydrogel beads and microspheres. Over the past few decades, chitosan beads have become important vehicles for use as potential drug delivery systems. Drug release rate can be controlled depending on the particle size, swelling ratio and change in environment such as $\mathrm{pH}$, temperature etc of the prepared beads. Recently, many attempts have been made to develop chitosan beads for the delivery of drugs, such as anticancer drugs, peptides, antibiotic agents or steroids etc. Because chitosan is easily soluble in acid, crosslinking of chitosan to form a network is the only way to prepare chitosan interpenetrating polymer networks (IPNs) (Mi et al 2000). Conventional chitosan crosslinking reactions have involved a reaction of chitosan with dialdehydes, such as glutaraldehyde.

*Author for correspondence (ppk923@yahoo.com)
L-glutamic acid is a non-essential, acidic and genetically coded amino acid. It has longer, slightly more flexible side chain having an acidic carboxyl group on it which can serve both as an acceptor and a donor of ammonia. Glutamic acid coupled with ammonia is called glutamine, the monoamide form of glutamic acid. Glutamic acid, most important digestive enzyme, is an abundant constituent of proteins. Besides glucose, glutamic acid is the only compound used for brain fuel. The brain converts glutamic acid to a compound that regulates brain cell activity (Grossfeld et al 1984). Glutamic acid increases the firing of neurons in the nervous system. It metabolizes sugars and fats, and detoxifies ammonia when used with glutamine (Doolittle 1989). It also helps correct personality disorders, including autism. It is also an important source that supplies hydrochloric acid to the digestive system which is used as a gastric acidifier (David and Michael 2000).

The purpose of the present study is to find out the effect of weight ratio of chitosan-glutamic acid, concentration of crosslinker and drug loading on swelling and release rates. Further the swelling and drug release data are utilized to propose the possible kinetics of swelling and drug release behaviours.

\section{Experimental}

\subsection{Materials}

Chitosan (percentage of deacetylation after drying: $80 \%$, total nitrogen: $7 \%$ minimum, loss on drying: $<15 \%$ and ignition residue (sulfate): $<2 \%$ ) was purchased from Tokyo 
Kasei Kogyo Co., Ltd., Japan and was used as received. Chlorpheniramine maleate (CPM) $\left[\mathrm{C}_{16} \mathrm{H}_{19} \mathrm{ClN}_{2} \mathrm{C}_{4} \mathrm{H}_{4} \mathrm{O}_{4}\right]$ was obtained as a gift sample from Japson Pharmaceuticals Ltd., Sangrur, India. Glutaraldehyde $\left(\mathrm{C}_{5} \mathrm{H}_{8} \mathrm{O}_{2}\right)(\mathrm{MW}=$ 100.11), acetic acid and glutamic acid $\left(\mathrm{C}_{5} \mathrm{H}_{9} \mathrm{NO}_{4}\right)$ $(\mathrm{MW}=147 \cdot 13)$ were procured from $\mathrm{CDH}$, New Delhi, India.

\subsection{Experimental design}

The study was carried out in two different steps. In the first step, the effect of concentration of crosslinker $(25 \%$, $12.5 \%, 6.25 \%$ and $3.13 \%$ ) on drug release behaviour was studied. In the second step, the drug release behaviour was studied while varying the ratio of chitosan and glutamic acid, keeping the amount of crosslinker as constant.

\subsection{Preparation of semi-interpenetrating network (semi-IPN) of chitosan and glutamic acid}

Purified chitosan and glutamic acid were dissolved in $2 \%$ acetic acid solution by stirring for $3 \mathrm{~h}$ at room temperature. The homogeneous mixture was extruded in the form of droplets, through a syringe of $0.56 \mathrm{~mm}$ diameter, into sodium hydroxide-methanol solution $(1: 20 \mathrm{w} / \mathrm{w})$ under stirring. The beads were washed thrice with hot $\left(50^{\circ} \mathrm{C}\right)$ and cold $\left(25^{\circ} \mathrm{C}\right)$ water and it generally took two to three min, respectively. The resultant beads were allowed to react with crosslinker at $50^{\circ} \mathrm{C}$ for about $10 \mathrm{~min}$ for crosslinking. Finally, the crosslinked beads were successively washed with hot $\left(50^{\circ} \mathrm{C}\right)$ and cold $\left(25^{\circ} \mathrm{C}\right)$ water, respectively and vacuum dried for $30 \mathrm{~min}$ at $-700 \mathrm{mmHg}$ and $55^{\circ} \mathrm{C}$. The mechanism of formation of crosslinked chitosan-chitosan and chitosan-glutamic acid IPN is shown in scheme 1 . The composition of the prepared beads is given in table 1.

To prepare the drug loaded beads, a known amount of CPM $(75,100$ and $150 \mathrm{mg}$, respectively) was added to the chitosan-glutamic acid mixture before extruding into the sodium hydroxide-methanol solution.

\subsection{Morphological characterization}

A JEOL JSM-6100 scanning electron microscope was used to perform textural characterization of chitosanglutamic acid beads. Each bead was fractured cryogenically and submitted to gold sputtering before analysis. Magnifications of $300 \mathrm{X}-1500 \mathrm{X}$ were applied to each sample in order to estimate the morphology.

\subsection{Swelling of semi-IPN of chitosan and glutamic acid}

To understand the molecular transport of liquid into semiIPN beads, dynamic swelling studies were performed in solutions of $\mathrm{pH} 2$ and $7 \cdot 4$, respectively. The dry samples of chitosan-glutamic acid beads (G1-G6) were soaked in alkaline and basic mediums at $37^{\circ} \mathrm{C}$. At different time intervals, beads were taken out and blotted off carefully in between tissue paper (without pressing hard) to remove the surface adhered solution. The swollen beads were then weighed $\left(W_{\mathrm{t}}\right)$ on the electronic microbalance (Shimadzu, A100X) to an accuracy of $\pm 0.01 \mathrm{mg}$. The percentage of swelling for each sample at time, $t$, is calculated using the relationship

$$
\text { Percentage of swelling }=\frac{W_{\mathrm{t}}-W_{\mathrm{o}}}{W_{\mathrm{o}}} \times 100,
$$

where $W_{\mathrm{o}}$ and $W_{\mathrm{t}}$ are the weights of the beads before and after swelling, respectively.

\subsection{Release of drug from semi-IPN of chitosan and glutamic acid}

The drug release experiments were performed in a glass apparatus at $37^{\circ} \mathrm{C}$ under unstirred conditions in acidic

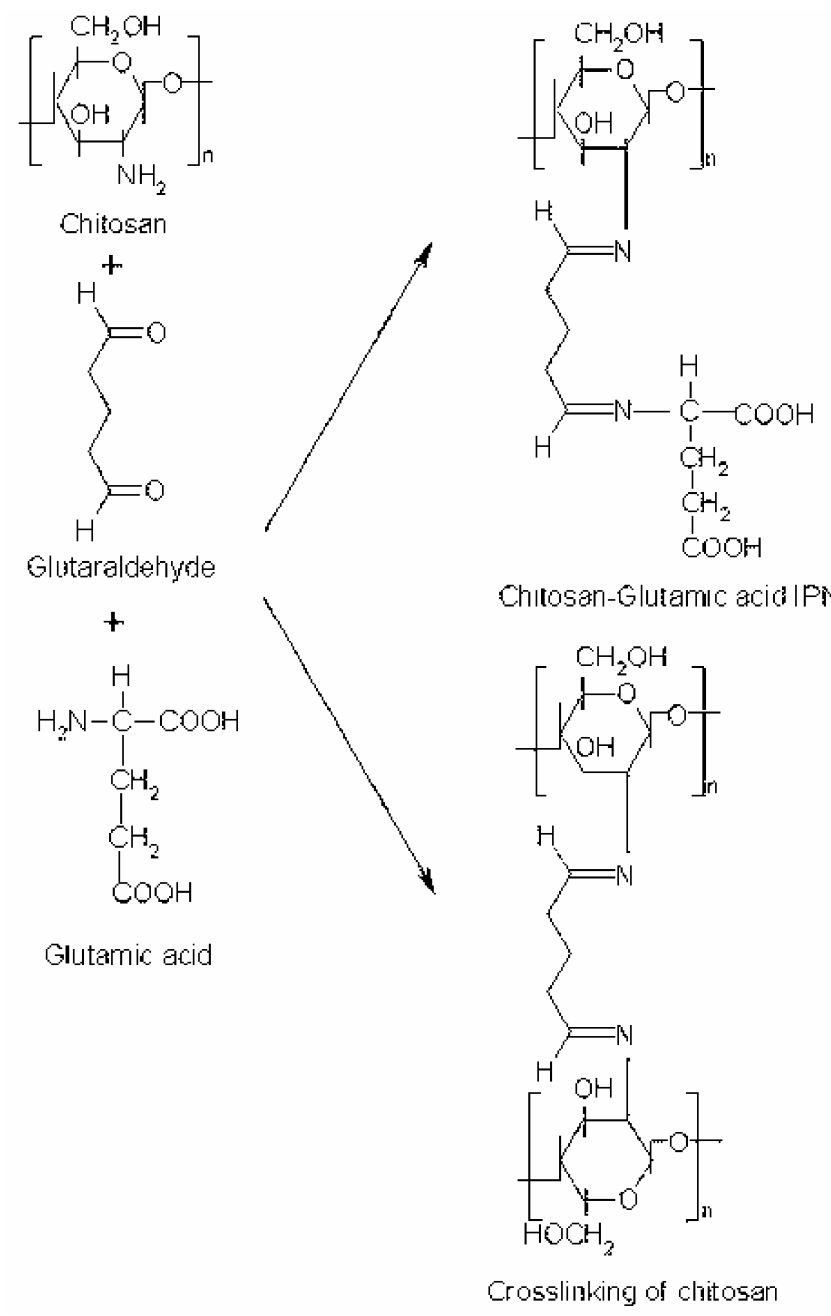

Scheme 1. Crosslinking of chitosan and glutamic acid by glutaraldehyde for the formation of semi-interpenetrating network (semi-IPN). 
Table 1. Composition and designation of chitosan-glutamic acid beads.

\begin{tabular}{|c|c|c|c|c|}
\hline Sample no* & Chitosan (g) & Glutamic acid (g) & $2 \%$ acetic acid $(\mathrm{ml})$ & Glutaraldehyde, $10 \mathrm{ml}(\%)$ \\
\hline G1 & $0 \cdot 5$ & $0 \cdot 5$ & 20 & $25 \cdot 00$ \\
\hline G2 & $0 \cdot 5$ & $0 \cdot 5$ & 20 & $12 \cdot 50$ \\
\hline G3 & $0 \cdot 5$ & 0.5 & 20 & $06 \cdot 25$ \\
\hline G4 & 0.5 & $0 \cdot 5$ & 20 & $03 \cdot 13$ \\
\hline G5 & 0.4 & 0.5 & 20 & $12 \cdot 50$ \\
\hline G6 & $0 \cdot 5$ & 0.4 & 20 & $12 \cdot 50$ \\
\hline
\end{tabular}

*These samples are further categorized as G11-G61, G12-G62 and G13-G63 to show the effect of drug loading. Where first digit followed by $\mathrm{G}$ indicates the sample number and second digit $(1,2$ and 3 ) stands for the amount of drug loading (15,20 and $30 \mathrm{mg})$, respectively.

$(\mathrm{pH} 2)$ and basic $(\mathrm{pH} \mathrm{7.4)}$ solutions. Beads $(0 \cdot 2 \mathrm{~g})$ containing known amounts of the drug were added to the release medium $(30 \mathrm{ml})$. At predecided intervals, samples of $3 \mathrm{ml}$ were withdrawn, filtered and assessed by recording the absorbance at $193.5 \mathrm{~nm}$. In order to maintain nearly constant release environment, the samples withdrawn for recording the absorbance were then immediately added back to the release medium.

\subsection{Kinetic analysis of swelling and drug release}

In order to have an insight into the mechanism of swelling and the drug release behaviour of the crosslinked beads, the power law equation and Higuchi's model were fitted into the kinetic data of swelling and drug release. Power law model (Korsmeyer et al 1983) (known as KorsmeyerPeppas equation) can be expressed as

$$
\frac{M_{\mathrm{t}}}{M_{\infty}}=k t^{n}
$$

According to Higuchi's (1963) model, an inert matrix should provide a sustained drug release over a reasonable period of time and yield a reproducible straight line when the fraction of drug released is plotted versus the square root of time.

$$
\frac{M_{\mathrm{t}}}{M_{\infty}}=k t^{1 / 2} .
$$

In case of swelling studies, $M_{\mathrm{t}}$ is the amount of solution absorbed at time, $t$ and $M_{\infty}$ the amount of solution absorbed at equilibrium. In case of release studies, $M_{\mathrm{t}}$ corresponds to the amount of drug release in time, $t, M_{\infty}$ the total amount of drug released after an infinite time, $k$ a constant related to the structural and geometric properties of the drug release system and $n$ the diffusional exponent. The numerical value of $n$ provides information about the mass transport mechanism for both the cases (swelling as well as release studies). When $n<0 \cdot 5$, the solvent diffuses through and the drug is released from the spherical polymeric matrix with a quasi-Fickian diffusion mechanism. An anomalous, non-Fickian drug diffusion occurs when the value of $n$ lies between 0.5 and 1 . If $n \geq 1$, a non-Fickian, case II or zero-order release kinetics can be observed.

\section{Results and discussion}

\subsection{SEM studies}

Crosslinked chitosan-glutamic acid beads prepared by using different concentrations of glutaraldehyde have rough and rigid surfaces. It is observed that the shape of the beads, as shown in figure 1a, was nearly spherical for samples G1-G4 and it changed to exact sphere in case of G5 and G6. This may be due to different compositions of the crosslinked beads (G5 and G6) as compared to G1-G4 beads. As the concentration of chitosan decreases as in the case of G5, the viscosity of the solution decreases and it becomes easier to extrude the spherical beads through a syringe. The same is true in case of G6, where the concentration of glutamic acid is least among all compositions. The approximate size of the beads is in the range of 1$1.2 \mathrm{~mm}$.

From the morphology of the beads as shown in figure $1 b$, one can find the rubbery, fibrous and folded surfaces of the beads. With higher concentration of crosslinker, as in case of G1, the chains come closer to each other and exhibit a regular, fibrous structure but with decreasing degree of crosslinker, as in case of G2 and G3, the structural morphology changes to layered and big fibrous bunches. Rubbery morphology is observed in case of lowest percentage of crosslinker (G4). The coil type morphology of G5 beads is different from the morphology of other beads which may be due to the lesser concentration of chitosan. Fibrous morphology of G6 beads is probably due to higher concentration of chitosan in the composition. It is observed that the morphology of G6 beads is more regular than G2, which may be attributed to the smaller concentration of a spacer i.e. glutamic acid. As a result of this, the chains come closer to each other despite having the same degree of crosslinker (12.5\%).

\subsection{Swelling studies}

Swelling response of the glutaraldehyde-crosslinked chitosan-glutamic acid beads in solutions of $\mathrm{pH} 2$ and 7.4 is shown in figure 2(a). It is observed that the swelling rate of the crosslinked beads having equal weight ratios 

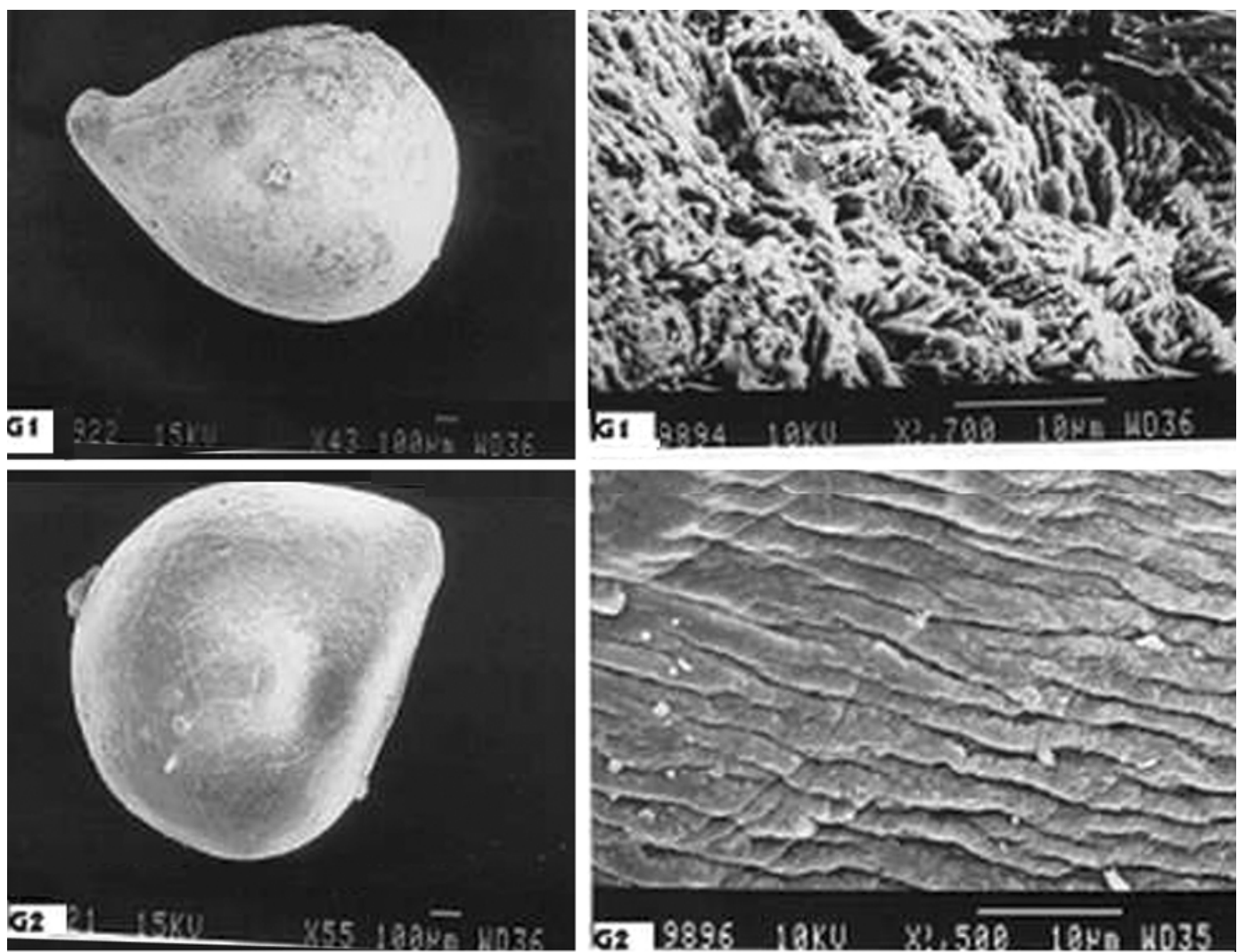

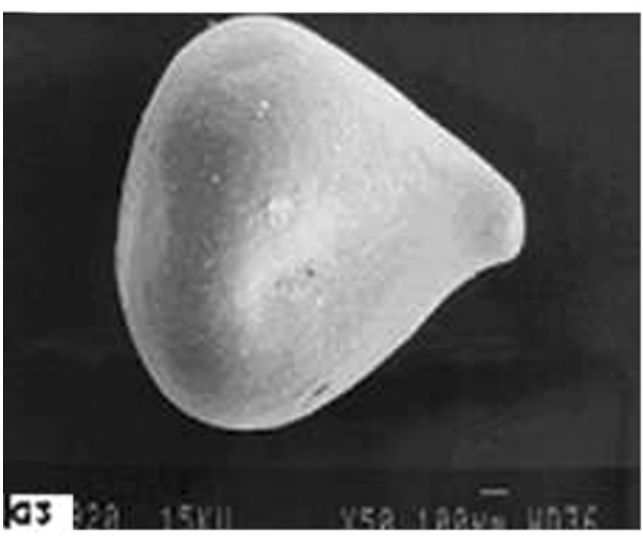

(a)

Figure 1. For caption, see next page.

of chitosan and glutamic acid with varying concentrations of crosslinker follows the order $\mathrm{G} 4>\mathrm{G} 3>\mathrm{G} 2>\mathrm{G} 1$. When the crosslinked beads are placed in the solution, the solution penetrates into the beads and the beads subsequently try to swell. Generally, the swelling process of the beads in $\mathrm{pH}<6$ involves the protonation of amino/imine groups in the beads and subsequent relaxation of the coiled polymeric chains. Initially, during the process of protonation, amino/imine groups of the bead surface are protonized which led to dissociation of the hydrogen bonding between amino/imine groups and other groups. Afterwards, protons and counterions diffuse into the bead

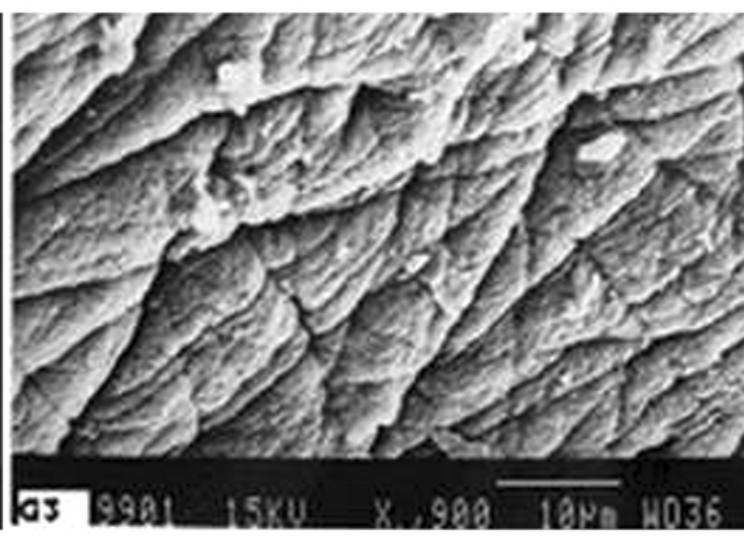

(b) to protonate the amino/imine groups inside the beads and dissociating the hydrogen bonds (Gupta and Kumar 2000, 2001).

It is observed that the swelling rates are directly proportional to the degree of crosslinking. As the higher crosslink density results in higher strength of the beads and lower degree of swelling, thus the lowest swelling rate is observed in case of G1 beads. Further, the percentage of swelling is higher in basic solution than in acidic solution. This may be due to the presence of free carboxylic ends of the chitosan-glutamic acid semi-IPN, which are more likely to be attacked by basic solution. The au- 

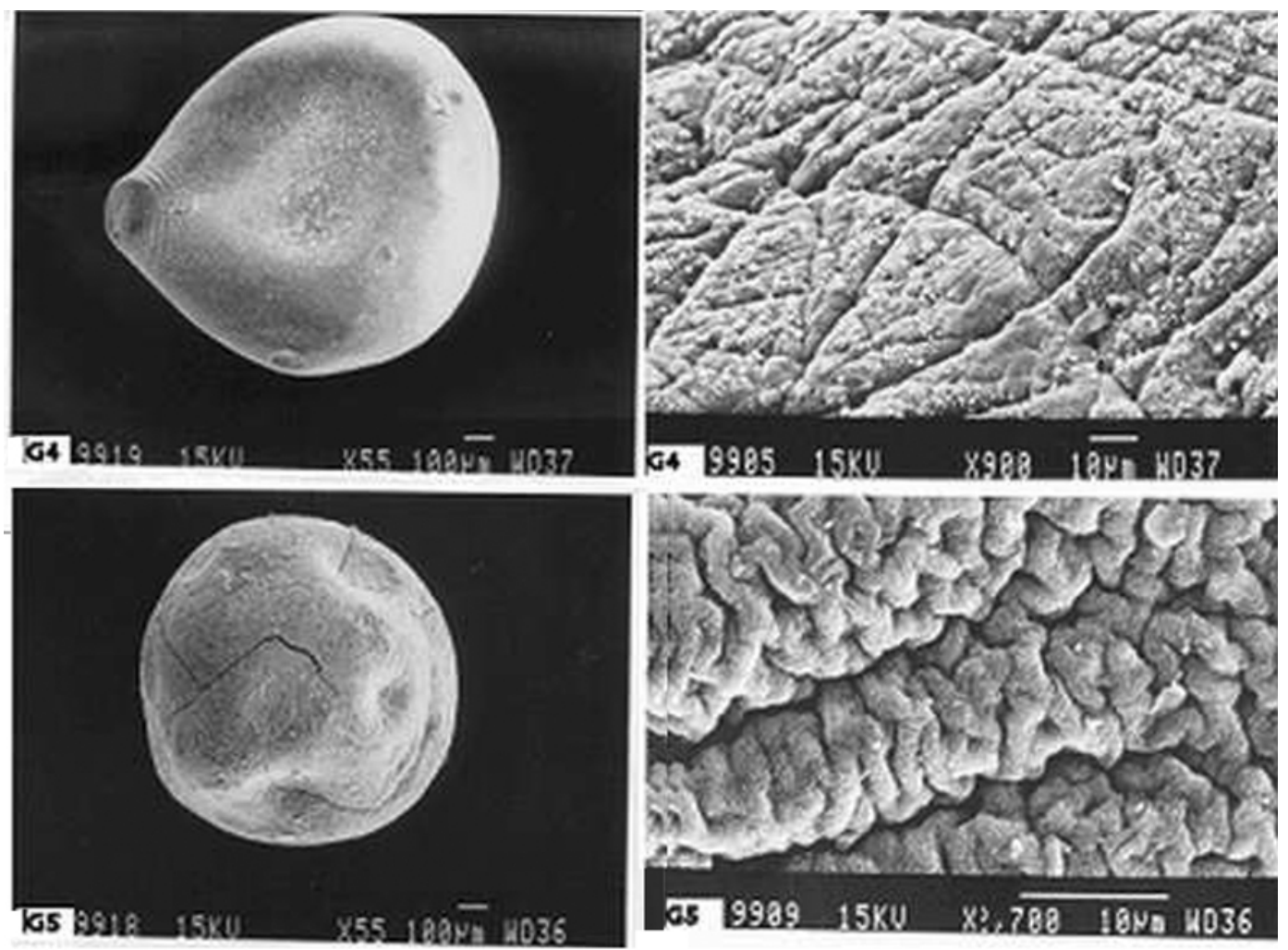

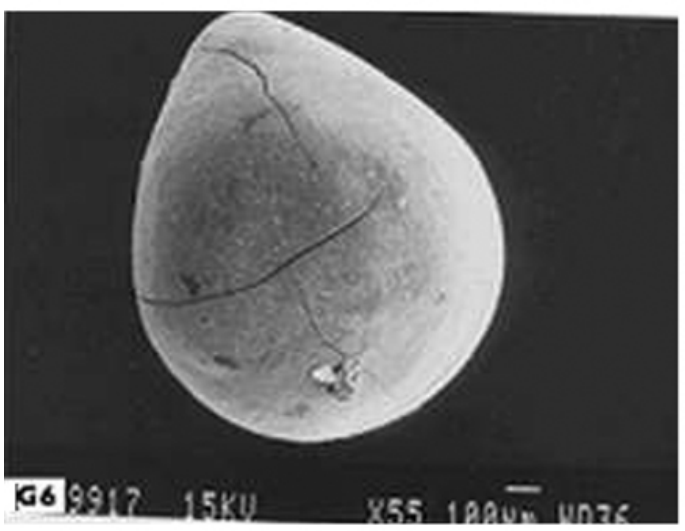

(a)

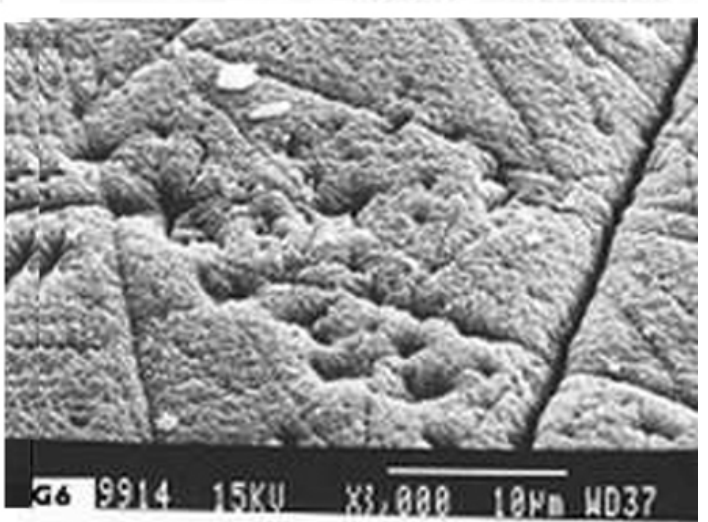

(b)

Figure 1. (a) SEM micrographs of the crosslinked beads (samples G1-G6) and (b) their morphology.

thors have performed similar studies for chitosan-alanine system (Kumari and Kundu 2007). It is observed that the percentage of swelling of chitosan-glutamic acid beads is slightly less as compared to the percentage of swelling of chitosan-alanine system having same composition.

The percentage of swelling of the crosslinked beads having the same concentration of crosslinker decreases with increasing concentration of chitosan (G5>G2>G6) and is shown in figure 2(b). It can be explained as the percentage of glutamic acid (which acts as a spacer) decreases, the pore size of the beads decreases and the penetration of the $\mathrm{pH}$ solution into the beads becomes difficult, resulting in lesser degree of swelling.

\subsection{Drug release studies}

To understand the release of CPM from the crosslinked chitosan-glutamic acid beads, the in vitro release experiments are performed with $0.2 \mathrm{~g}$ of sample containing $15 \mathrm{mg}, 20 \mathrm{mg}$ and $30 \mathrm{mg}$ of drug, respectively. The dissolution profile of chlorpheniramine maleate (CPM) from crosslinked beads at various time intervals in acidic $(\mathrm{pH}$ 2) and basic ( $\mathrm{pH} \mathrm{7.4)} \mathrm{mediums} \mathrm{is} \mathrm{shown} \mathrm{in} \mathrm{figures} \mathrm{3-5.} \mathrm{A}$ burst release is observed, initially for the first hour in both media followed by a moderate release for next five hours and finally an almost constant release of CPM from the matrix is observed for the studied period of $48 \mathrm{~h}$. The 
drug release rate is found to increase with the decrease in crosslink density. This may be due to the fact that the diffusion of drug from the semi-IPN depends on the pore size of the polymer network, which will decrease with increase in degree of crosslinking (Kumar and Gupta 2000; Kumari and Kundu 2007).

The dissolution profiles of chlorpheniramine maleate (CPM) from the crosslinked chitosan-glutamic acid beads at various time intervals indicate that the amount and percentage of drug released is much higher in basic solution than in acidic solution. This can be explained by the fact that the release of drug depends mainly on the percentage of swelling of beads. Initially, the burst release of drug is observed due to the fast penetration of the solvent into the crosslinked beads, which is governed by the Fick's law of diffusion. After few hours, a steady state is reached leading to a plateau nature of plots. A very small change in the concentration of drug released is observed during $24-48 \mathrm{~h}$ period. After $48 \mathrm{~h}$, no significant change in concentration of drug release was observed from the

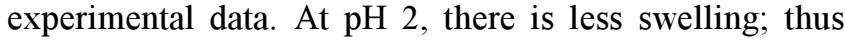
drug entrapped in the beads cannot be released easily. However, at $\mathrm{pH} 7 \cdot 4$, the beads are swollen to a higher percentage, leading to faster release of drug. The higher rate of drug release in the basic medium may be due to the presence of two carboxylic ends (scheme 1) of the crosslinked chitosan-glutamic acid semi-IPN. Since, carboxylic group is more susceptible to attack by the basic solution, the drug release in the acidic medium is less due to less interaction of acidic solution with the polar groups of semi-IPN.

According to the earlier studies, the amount and percentage of drug released in $\mathrm{pH} 7.4$ is higher in case of chitosan-alanine system, whereas, it is slightly lower in $\mathrm{pH} 7.4$ for the present system of chitosan-glutamic acid. This may be due to the better compatibility of aliphatic end of CPM with glutamic acid because of the presence of long chain of hydrocarbon in it.
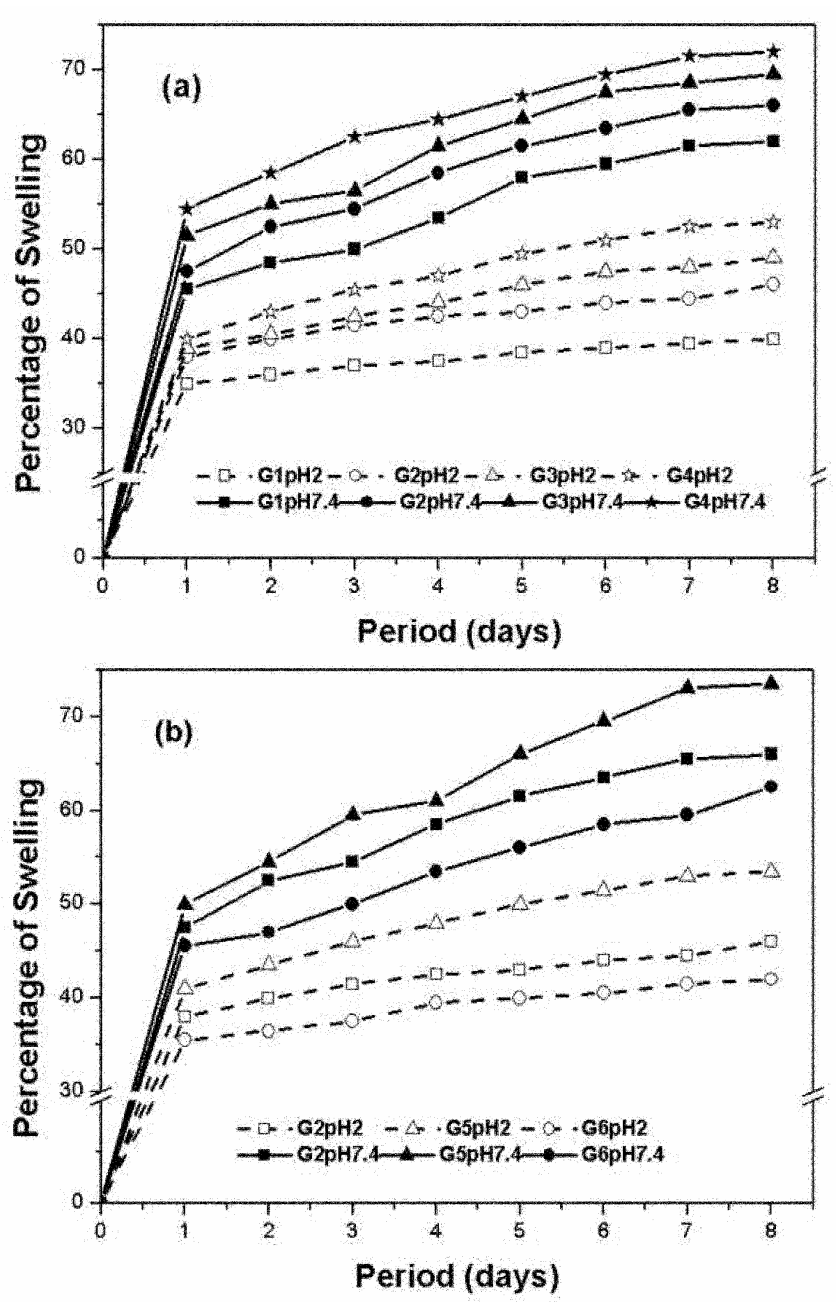

Figure 2. Swelling behaviour of the crosslinked beads measured as a function of time in $\mathrm{pH} 2$ and 7.4: (a) having same and (b) different weight ratios of chitosan and glutamic acid.
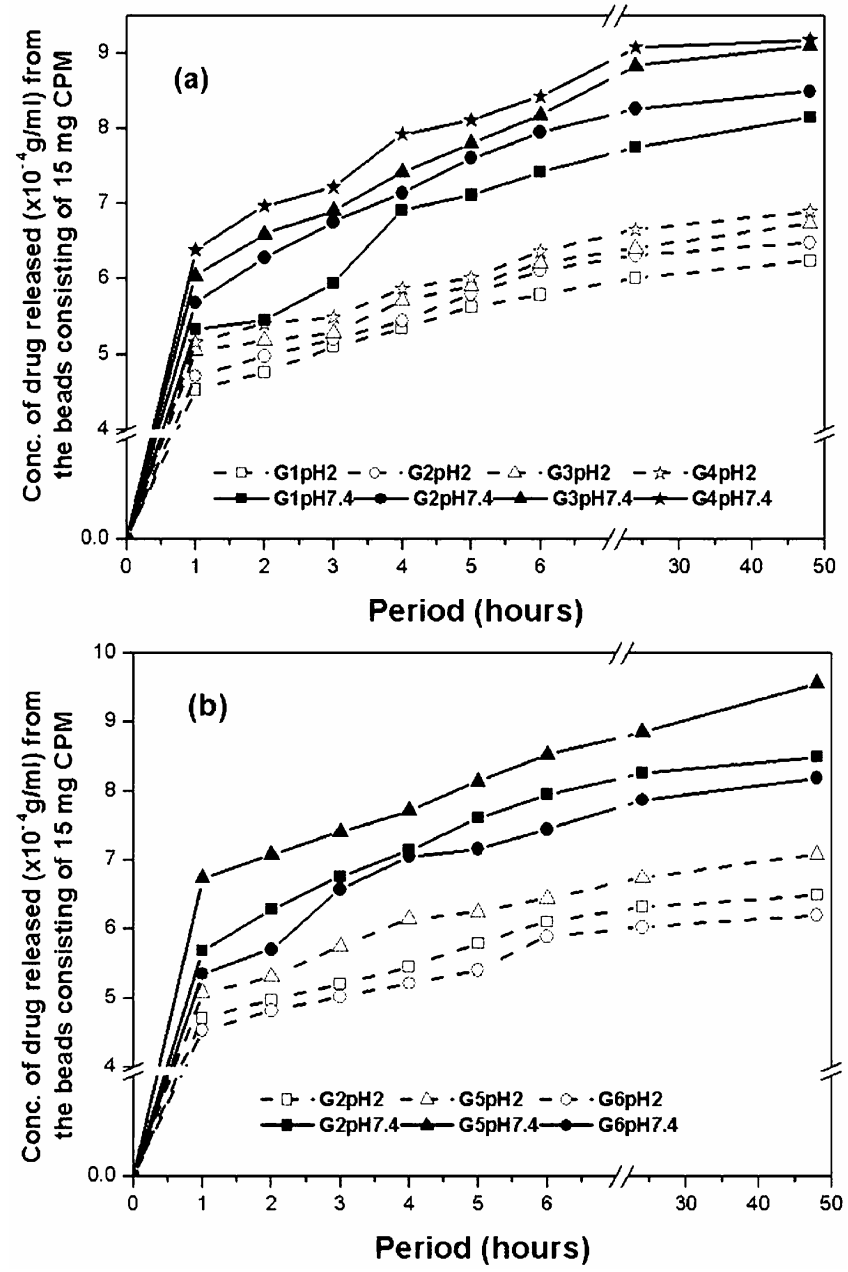

Figure 3. Release of CPM (15 mg) loaded beads vs time in solutions of $\mathrm{pH} 2$ and 7.4: (a) having same and (b) different weight ratios of chitosan and glutamic acid. 
In the second step of studies, the release experiments are performed for the beads with varying weight ratios of chitosan and glutamic acid having the same percentage of crosslinker (i.e. $12 \cdot 5 \%$ ). It is observed that the beads having lower concentration of chitosan have higher release rate, followed by the equal weight ratios of chitosan and glutamic acid. The slowest release rate is observed in case of higher concentration of chitosan. The lower concentration of glutamic acid (spacer) induces a closer association of chitosan chains, leading to a decreasing rate of swelling as well as drug release.

The drug release also shows a dependence on the amount of drug loading. The maximum amount of drug released from $0.2 \mathrm{~g}$ of sample containing $15 \mathrm{mg}$ CPM is $9.56 \times$ $10^{-4} \mathrm{~g} \cdot \mathrm{ml}^{-1}$ in basic and $7.08 \times 10^{-4} \mathrm{~g} \cdot \mathrm{ml}^{-1}$ in acidic solution and the lower limit of drug released from the same composition is $8.18 \times 10^{-4} \mathrm{~g} \cdot \mathrm{ml}^{-1}$ in basic and $6 \cdot 1 \times$ $10^{-4} \mathrm{~g} \cdot \mathrm{ml}^{-1}$ in acidic medium. The maximum amount of drug released increases to $11.4 \times 10^{-4} \mathrm{~g} \cdot \mathrm{ml}^{-1}$ in basic and $8 \cdot 14 \times 10^{-4} \mathrm{~g} \cdot \mathrm{ml}^{-1}$ in acidic solutions in case of $30 \mathrm{mg}$
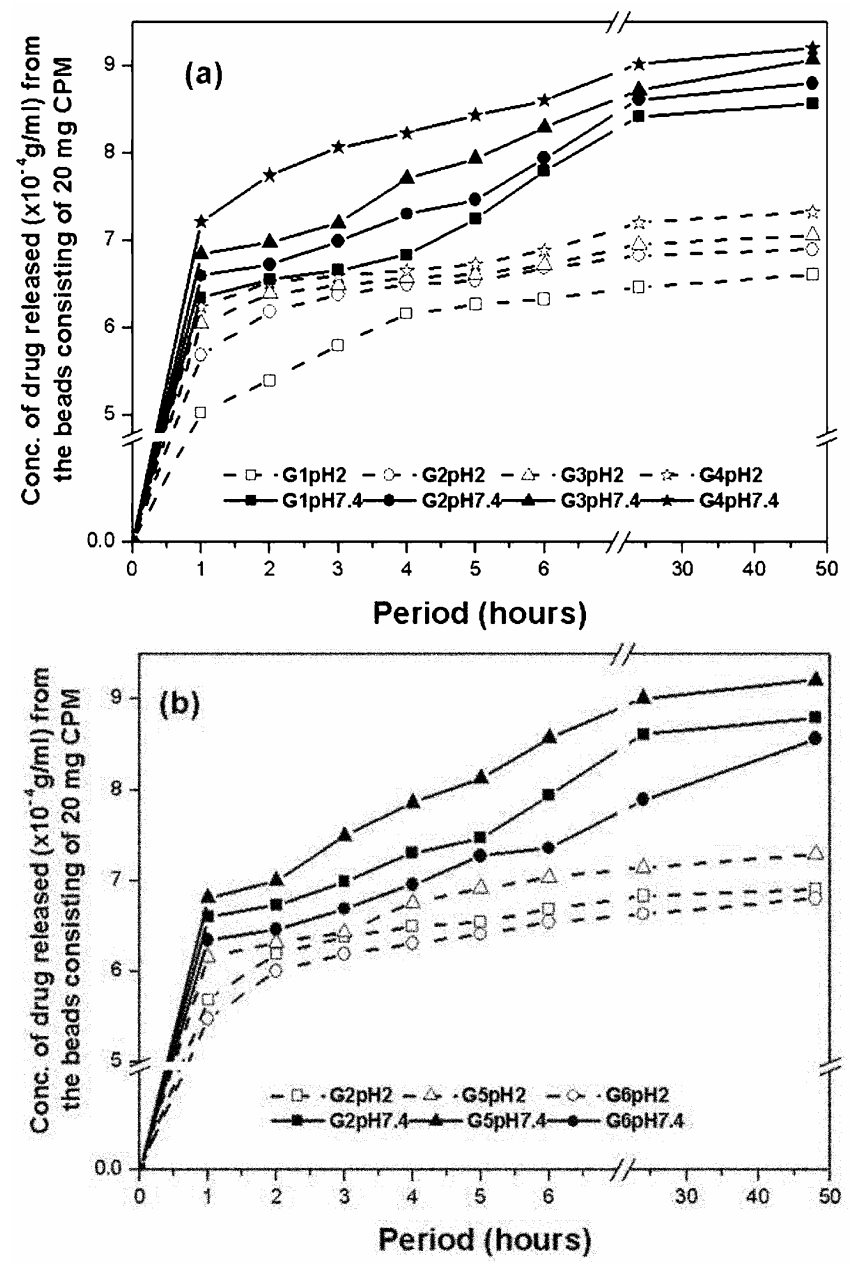

Figure 4. Release of CPM (20 mg) loaded beads vs time in solutions of $\mathrm{pH} 2$ and 7.4: (a) having same and (b) different weight ratios of chitosan and glutamic acid.
CPM loaded beads and the minimum amount of drug released is $10.4 \times 10^{-4} \mathrm{~g} \cdot \mathrm{ml}^{-1}$ and $7.06 \times 10^{-4} \mathrm{~g} \cdot \mathrm{ml}^{-1}$ in the basic and acidic mediums, respectively. It is observed that the release is faster in case of $30 \mathrm{mg}$ CPM loaded beads as compared to $20 \mathrm{mg}$ and $15 \mathrm{mg}$ drug loaded beads. This may be explained that due to the higher concentration gradient, there is higher rate of molecular diffusion and thus more the release of drug. Hence, the maximum amount of drug released after prolonged time approaches the maximum drug present in the sample (e.g. $9.56 \times 10^{-4} \mathrm{~g} \cdot \mathrm{ml}^{-1}$ from $15 \mathrm{mg}$ drug loaded beads). The swelling experiments indicate that the solvent will penetrate into the drug loaded samples.

\subsection{Kinetic analysis of swelling and drug release}

Power law equation (2) and Higuchi's model (3) are used to analyse the dynamic swelling behaviour of the crosslinked beads and the drug release mechanism. The drug
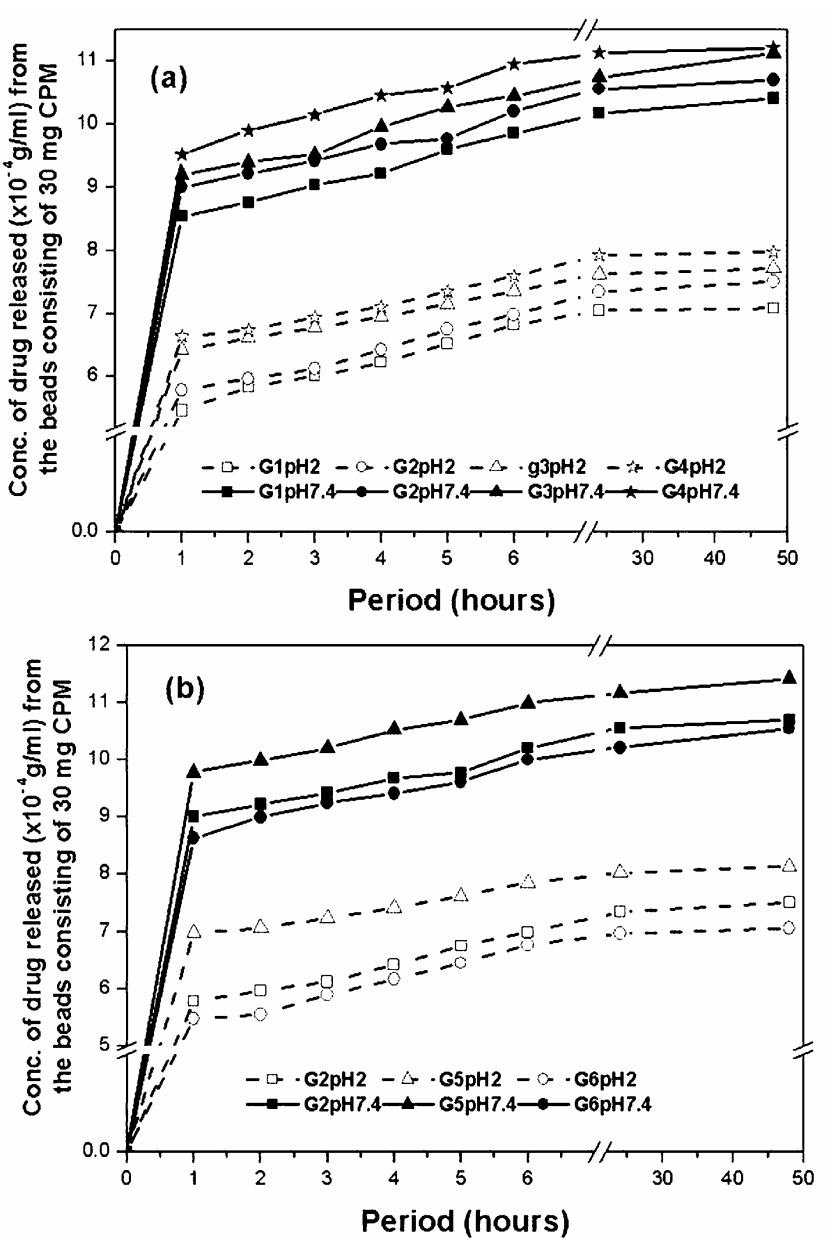

Figure 5. Release of CPM (30 mg) loaded beads vs time in solutions of $\mathrm{pH} 2$ and 7.4: (a) having same and (b) different weight ratios of chitosan and glutamic acid. 
Table 2. Results of swelling mechanism calculated from (2) and (3).

\begin{tabular}{|c|c|c|c|c|c|c|c|c|c|c|c|c|c|c|}
\hline \multirow[b]{3}{*}{ Formulation } & \multicolumn{7}{|c|}{$\mathrm{pH}=7 \cdot 4$} & \multicolumn{7}{|c|}{$\mathrm{pH}=2 \cdot 0$} \\
\hline & \multicolumn{4}{|c|}{$\begin{array}{l}\text { Power law model } \\
\quad\left(M_{\mathrm{t}} / M_{\infty}=k t^{n}\right)\end{array}$} & \multicolumn{3}{|c|}{$\begin{array}{l}\text { Higuchi model } \\
\left(M_{\mathrm{t}} / M_{\infty}=k t^{1 / 2}\right)\end{array}$} & \multicolumn{4}{|c|}{$\begin{array}{l}\text { Power law model } \\
\quad\left(M_{\mathrm{t}} / M_{\infty}=k t^{n}\right)\end{array}$} & \multicolumn{3}{|c|}{$\begin{array}{l}\text { Higuchi model } \\
\left(M_{\mathrm{t}} / M_{\infty}=k t^{1 / 2}\right)\end{array}$} \\
\hline & $k$ & $n$ & S.D. & $R$ & $k$ & S.D. & $R$ & $k$ & $n$ & S.D. & $R$ & $k$ & S.D. & $R$ \\
\hline G1 & $0 \cdot 43$ & $0 \cdot 16$ & \pm 0.013 & $0 \cdot 97$ & 0.03 & \pm 0.018 & 0.99 & $0 \cdot 70$ & $0 \cdot 07$ & \pm 0.003 & 0.99 & $0 \cdot 01$ & $\pm 0 \cdot 002$ & 0.99 \\
\hline G2 & 0.42 & $0 \cdot 17$ & \pm 0.006 & 0.99 & 0.03 & \pm 0.011 & 0.99 & $0 \cdot 60$ & 0.09 & \pm 0.003 & 0.99 & 0.02 & \pm 0.006 & 0.99 \\
\hline G3 & $0 \cdot 44$ & $0 \cdot 16$ & \pm 0.011 & 0.98 & 0.03 & \pm 0.016 & 0.99 & $0 \cdot 54$ & $0 \cdot 11$ & \pm 0.007 & $0 \cdot 98$ & $0 \cdot 02$ & $\pm 0 \cdot 008$ & 0.99 \\
\hline G4 & $0 \cdot 48$ & $0 \cdot 14$ & \pm 0.005 & $0 \cdot 99$ & 0.03 & \pm 0.007 & 0.99 & $0 \cdot 68$ & $0 \cdot 14$ & \pm 0.005 & 0.99 & $0 \cdot 03$ & \pm 0.006 & 0.99 \\
\hline G5 & $0 \cdot 35$ & $0 \cdot 19$ & \pm 0.010 & 0.99 & $0 \cdot 04$ & \pm 0.013 & 0.99 & 0.49 & $0 \cdot 14$ & \pm 0.005 & $0 \cdot 99$ & 0.03 & \pm 0.006 & $0 \cdot 99$ \\
\hline G6 & $0 \cdot 42$ & $0 \cdot 16$ & \pm 0.013 & $0 \cdot 97$ & 0.03 & \pm 0.015 & 0.99 & $0 \cdot 63$ & $0 \cdot 08$ & \pm 0.006 & $0 \cdot 98$ & $0 \cdot 02$ & \pm 0.08 & 0.99 \\
\hline
\end{tabular}

Table 3. Results of drug release mechanism showing the effect of degree of crosslinking and amount of drug loaded calculated from (2) and (3).

\begin{tabular}{|c|c|c|c|c|c|c|c|c|c|c|c|c|c|c|}
\hline \multirow[b]{3}{*}{ Formulation } & \multicolumn{7}{|c|}{$\mathrm{pH}=7 \cdot 4$} & \multicolumn{7}{|c|}{$\mathrm{pH}=2 \cdot 0$} \\
\hline & \multicolumn{4}{|c|}{$\begin{array}{l}\text { Power law model } \\
\quad\left(M_{t} / M_{\infty}=k t^{n}\right)\end{array}$} & \multicolumn{3}{|c|}{$\begin{array}{l}\text { Higuchi model } \\
\left(M_{\mathrm{t}} / M_{\infty}=k t^{1 / 2}\right)\end{array}$} & \multicolumn{4}{|c|}{$\begin{array}{l}\text { Power law model } \\
\quad\left(M_{\mathrm{t}} / M_{\infty}=k t^{n}\right)\end{array}$} & \multicolumn{3}{|c|}{$\begin{array}{l}\text { Higuchi model } \\
\left(M_{t} / M_{\infty}=k t^{1 / 2}\right)\end{array}$} \\
\hline & $k$ & $n$ & S.D. & $R$ & $k$ & S.D. & $R$ & $k$ & $n$ & S.D. & $R$ & $k$ & S.D. & $R$ \\
\hline G11 & 0.62 & $0 \cdot 20$ & \pm 0.024 & 0.94 & $0 \cdot 19$ & \pm 0.033 & $0 \cdot 96$ & $0 \cdot 71$ & $0 \cdot 14$ & \pm 0.009 & $0 \cdot 98$ & $0 \cdot 15$ & \pm 0.009 & 0.99 \\
\hline G21 & 0.66 & $0 \cdot 19$ & \pm 0.007 & 0.99 & $0 \cdot 18$ & \pm 0.006 & 0.99 & 0.71 & $0 \cdot 14$ & \pm 0.013 & 0.96 & $0 \cdot 15$ & \pm 0.016 & 0.98 \\
\hline G31 & 0.65 & 0.67 & \pm 0.009 & 0.98 & $0 \cdot 16$ & \pm 0.009 & 0.99 & 0.73 & $0 \cdot 11$ & \pm 0.016 & 0.92 & $0 \cdot 12$ & \pm 0.022 & 0.96 \\
\hline G41 & 0.69 & $0 \cdot 16$ & \pm 0.009 & 0.98 & $0 \cdot 16$ & \pm 0.013 & 0.99 & 0.73 & $0 \cdot 11$ & \pm 0.012 & 0.95 & $0 \cdot 12$ & \pm 0.017 & 0.97 \\
\hline G51 & 0.69 & $0 \cdot 13$ & \pm 0.010 & 0.97 & $0 \cdot 13$ & \pm 0.013 & 0.99 & $0 \cdot 70$ & $0 \cdot 14$ & \pm 0.009 & 0.98 & $0 \cdot 14$ & \pm 0.014 & 0.98 \\
\hline G61 & $0 \cdot 64$ & $0 \cdot 20$ & \pm 0.013 & 0.98 & $0 \cdot 19$ & \pm 0.022 & 0.98 & 0.72 & $0 \cdot 13$ & \pm 0.014 & 0.95 & $0 \cdot 14$ & \pm 0.021 & 0.97 \\
\hline G12 & 0.73 & 0.07 & \pm 0.010 & 0.92 & $0 \cdot 11$ & \pm 0.027 & 0.92 & 0.76 & $0 \cdot 14$ & \pm 0.007 & 0.99 & $0 \cdot 15$ & \pm 0.015 & 0.99 \\
\hline G22 & 0.69 & $0 \cdot 14$ & \pm 0.007 & 0.97 & $0 \cdot 10$ & \pm 0.017 & $0 \cdot 96$ & $0 \cdot 83$ & 0.09 & \pm 0.005 & 0.98 & $0 \cdot 07$ & \pm 0.005 & 0.99 \\
\hline G32 & $0 \cdot 68$ & $0 \cdot 16$ & \pm 0.006 & 0.98 & $0 \cdot 11$ & \pm 0.018 & $0 \cdot 97$ & $0 \cdot 86$ & 0.06 & \pm 0.003 & 0.99 & $0 \cdot 06$ & \pm 0.009 & 0.97 \\
\hline G42 & 0.79 & 0.09 & \pm 0.001 & 0.99 & $0 \cdot 10$ & \pm 0.007 & $0 \cdot 99$ & $0 \cdot 85$ & 0.05 & \pm 0.003 & 0.98 & $0 \cdot 05$ & \pm 0.007 & 0.98 \\
\hline G52 & $0 \cdot 67$ & $0 \cdot 18$ & \pm 0.004 & 0.99 & $0 \cdot 13$ & \pm 0.015 & $0 \cdot 98$ & $0 \cdot 83$ & 0.08 & \pm 0.007 & $0 \cdot 96$ & $0 \cdot 09$ & \pm 0.010 & $0 \cdot 98$ \\
\hline G62 & $0 \cdot 69$ & $0 \cdot 13$ & \pm 0.014 & 0.99 & 0.09 & $\pm 0 \cdot 012$ & $0 \cdot 98$ & $0 \cdot 81$ & 0.09 & \pm 0.005 & 0.99 & $0 \cdot 10$ & \pm 0.015 & 0.97 \\
\hline G13 & $0 \cdot 81$ & 0.08 & \pm 0.008 & 0.95 & 0.09 & \pm 0.010 & $0 \cdot 98$ & 0.76 & $0 \cdot 12$ & \pm 0.009 & 0.97 & $0 \cdot 13$ & \pm 0.010 & 0.99 \\
\hline G23 & $0 \cdot 83$ & 0.07 & \pm 0.007 & 0.95 & 0.07 & \pm 0.010 & $0 \cdot 97$ & 0.75 & $0 \cdot 10$ & \pm 0.012 & $0 \cdot 94$ & $0 \cdot 11$ & \pm 0.060 & 0.97 \\
\hline G33 & $0 \cdot 81$ & 0.07 & \pm 0.008 & 0.94 & 0.08 & \pm 0.014 & $0 \cdot 96$ & $0 \cdot 83$ & 0.07 & \pm 0.006 & 0.96 & $0 \cdot 08$ & \pm 0.008 & 0.99 \\
\hline G43 & $0 \cdot 84$ & 0.08 & \pm 0.004 & 0.98 & $0 \cdot 09$ & \pm 0.006 & 0.99 & $0 \cdot 81$ & 0.07 & \pm 0.009 & $0 \cdot 94$ & $0 \cdot 08$ & $\pm 0 \cdot 012$ & 0.97 \\
\hline G53 & $0 \cdot 85$ & 0.06 & \pm 0.006 & $0 \cdot 96$ & 0.07 & \pm 0.008 & $0 \cdot 98$ & $0 \cdot 84$ & 0.06 & \pm 0.008 & 0.93 & 0.07 & \pm 0.010 & 0.97 \\
\hline G63 & $0 \cdot 81$ & 0.08 & \pm 0.005 & 0.98 & 0.09 & \pm 0.008 & 0.99 & $0 \cdot 69$ & $0 \cdot 18$ & \pm 0.004 & 0.99 & $0 \cdot 13$ & \pm 0.020 & 0.97 \\
\hline
\end{tabular}

release through IPN beads depends on the percentage of swelling of the network. Several factors, such as the nature and composition of the IPN synthesized, govern the degree of swelling as well as drug release from the beads. Moreover, the nature of swelling and release environment is also an important contributing factor towards the percentage of swelling and drug release.

Swelling and drug release results are analysed using (2) and (3). The swelling and initial drug release data are plotted in Korsmeyer's equation (2) as log of cumulative percentage of swelling/drug released vs log of time. The values of $n$ and $k$ are determined by applying the linear regression method. The data for swelling and release mechanism for different formulations, along with the values of correlation coefficients, $R$, are presented in tables 2,3 , respectively. The value of $k$ for swelling as well as for release increases with a decrease in $\mathrm{pH}$ of the environment and the concentration of crosslinker. Moreover, $k$ value is found to be higher for the higher drug loaded samples. On the other hand, the value of diffusional exponent, $n$, decreases with an increase in the amount of drug loading in both the release environments. It means that the value of $n$ increases with an increase in crosslink density. For all the samples, the value of $n$ is less than $0 \cdot 5$, which implies that diffusional release mechanism is quasi-Fickian diffusion.

Higuchi's model (3) describes the release of drug from an insoluble matrix as square root of time-dependent process. The constant, $k$, is calculated from the slope of the plot of cumulative drug released vs the square root of time. The value of $k$, for the release process, ranges between $0 \cdot 07-0 \cdot 19$ and $0 \cdot 01-0 \cdot 15$ in solutions of $\mathrm{pH} 7 \cdot 4$ and 
2 , respectively. The smaller values of $k$ indicate mild interaction between the drug and IPN matrices (Agnihotri and Aminabhavi 2006).

\section{Conclusions}

Chitosan-glutamic acid beads are prepared and crosslinked with glutaraldehyde to form semi-IPNs in two ways. In the first process, the beads are prepared having the same weight ratio but varying amounts of crosslinker whereas in the second process, the beads of different weight ratios are prepared with fixed amount of crosslinker. The morphological studies of the samples are carried out by using a scanning electron microscope (SEM). The swelling of beads and release rate of chlorpheniramine maleate (CPM) in different $\mathrm{pH}$ solutions are studied. It is observed that the release of CPM is more in basic medium than in acidic medium due to higher degree of swelling. From these investigations, it is evident that the rate of swelling of matrix and release of drugs is dependent on the degree of crosslinking, weight ratio of chitosanglutamic acid and the $\mathrm{pH}$ of solution. Therefore, by varying the weight ratio of chitosan-glutamic acid, concentration of glutaraldehyde and drug loading, the desired release rates can be achieved. Further, it also helps in optimizing drug entrapping capacity and its sustained release for an extended period of time. The results suggest that chitosan- glutamic acid crosslinked beads are suitable for controlled release of drug.

\section{References}

Agnihotri S A and Aminabhavi T M 2006 Int. J. Pharm. 324103

David L N and Michael M C 2000 Lehninger's principles of biochemistry (Worth Publishers) 3rd ed.

Doolittle R F 1989 Redundancies in protein sequences in prediction of protein structure and the principles of protein conformation (ed.) G D Fasman (New York: Plenum Press) p. 599

Grossfeld R M, Yancey S W and Baxter C F 1984 Comp. Biochem. Physiol. B78 287

Gupta K C and Kumar M N V R 2000 Biomaterials 211115

Gupta K C and Kumar M N V R 2001 J. Appl. Polym. Sci. 80639 Higuchi T 1963 J. Pharm. Sci. 521145

Ko J A, Park H J, Hwang S J, Park J B and Lee J S 2002 Int. J. Pharm. 249165

Korsmeyer R W, Gurny R, Doelker E, Buri P and Peppas N A 1983 Int. J. Pharm. 1525

Kumar M N V R and Gupta K C 2000 J. Appl. Polym. Sci. 76672

Kumari K and Kundu P P 2007 J. Appl. Polym. Sci. 1033751

Mi F L, Sung H W and Shyu S S 2000 J. Polym. Sci., Part A: Polym. Chem. 382804

Muzzarelli R A 1985 in The polysaccharides (ed.) G O A Aspinall (New York: Academic Press) p. 417

Thanoo B C, Sunny M C and Jayakrishnan A 1992 J. Pharm. Pharmacol. 44283 